\title{
Tratamento cirúrgico da tetralogia de Fallot no primeiro ano de vida
}

\author{
Fernando MORAES NETO*, Cláudio A. GOMES*, Cleusa LAPA*, Sheila HAZIN*, Euclides TENÓRIO*, \\ Sandra MATTOS* ${ }^{*}$ Carlos R. MORAES*
}

RBCCV 44205-496

Moraes Neto F, Gomes C A, Lapa C, Hazin S, Tenório E, Mattos S, Moraes C R - Tratamento cirúrgico da tetralogia de Fallot no primeiro ano de vida. Rev Bras Cir Cardiovasc 2000; 15 (2): 143-53.

RESUMO: Objetivo: Analisar os resultados do tratamento cirúrgico da tetralogia de Fallot (TF) no primeiro ano de vida, procurando especialmente definir as eventuais vantagens da correção definitiva precoce.

Casuística e Métodos: Entre março de 1986 e setembro de 1999, 56 crianças com menos de um ano de idade portadoras de tetralogia de Fallot foram submetidas a tratamento cirúrgico. Trinta e seis eram do sexo masculino e 20 do feminino, variando a idade de um a 11 meses (média 6,5 meses). O peso variou de 3 a $10 \mathrm{~kg}$ (média 6,3 kg). Esses pacientes foram divididos em dois grupos de acordo com o tipo de operação realizada: Grupo I: composto de 26 crianças operadas entre março de 86 e março de 96, submetidas a operação de Blalock-Taussig; Grupo II: formado por 30 crianças, operadas a partir de 1996, submetidas a correção definitiva com circulação extracorpórea (CEC).

Resultados: No Grupo I ocorreram 2 (7,6\%) óbitos imediatos e 1 (3,8\%) tardio após outra operação de Blalock. Nos casos já submetidos acorreção intracardíaca, a presença do shunt não determinou complicações e a incidência de ampliação do anel foi de $66,6 \%$. No Grupo II ocorreram $2(6,6 \%)$ óbitos imediatos e 1 (3,3\%) tardio de causa não cardíaca. A ampliação transanular da via de saída do ventrículo direito (VD) foi necessária em $50 \%$ dos casos. A evolução tardia desse grupo de doentes é excelente, todos estão assintomáticos, a função ventricular é normal e o gradiente na via de saída do VD varia de 10 a $38 \mathrm{mmHg}$ (média: $26 \mathrm{mmHg}$ ).

Conclusões: $O$ tratamento ideal da Tetralogia de Fallot no primeiro ano de vida é a correção definitiva, visto que o risco cirúrgico é igual ao da paliação. Ademais, a correção total restaura precocemente a fisiologia normal do coração e da circulação e a saturação arterial de oxigênio. Existem ainda evidentes vantagens sócio-econômicas e psicológicas.

DESCRITORES: Tetralogia de Fallot, cirurgia, lactente. Tetralogia de Fallot, cirurgia. Cirurgia cardíaca, pediátrica.

\section{INTRODUÇÃO}

O manuseio cirúrgico da tetralogia de Fallot (TF) no primeiro ano de vida é um assunto controverso, existindo ainda os que advogam a correção em dois tempos e os que indicam a correção primária precoce. Em nossa Instituição adotamos, a partir de 1996, a conduta de realizar a correção total no primeiro ano de vida em todos os casos de TF desde que não apresentem atresia pulmonar e/ou malformações associadas (Fallot clássico).

O objetivo do presente trabalho é analisar a morbi-mortalidade da cirurgia da TF no primeiro ano de vida, procurando especialmente definir as eventuais vantagens da correção definitiva precoce.

Trabalho realizado no Instituto do Coração de Pernambuco (Real Hospital Português de Beneficência em Pernambuco). Recife, PE, Brasil. Apresentado ao 27으 Congresso Nacional de Cirurgia Cardíaca. Rio de Janeiro, RJ, 23 a 25 de março, 2000.

*Do Instituto do Coração de Pernambuco.

Endereço para correspondência: Fernando Moraes Neto. Instituto do Coração de Pernambuco. Av. Portugal, 163. Recife, PE, Brasil. CEP 52010-010. Telefax: (081) 221-0382. e-mail: f_moraes@interway.com.br 
Moraes Neto F, Gomes C A, Lapa C, Hazin S, Tenório E, Mattos S, Moraes C R - Tratamento cirúrgico da tetralogia de Fallot no primeiro ano de vida. Rev Bras Cir Cardiovasc 2000; 15 (2): 143-53.

\section{CASUÍSTICA E MÉTODOS}

Entre março de 1986 e setembro de 1999, 56 crianças com menos de um ano de idade portadoras de TF foram submetidas a tratamento cirúrgico no Instituto do Coração de Pernambuco (Real Hospital Português). Trinta e seis $(64,3 \%)$ eram do sexo masculino e $20(35,7 \%)$ do feminino. A idade variou de um a 11 meses (média: 6,5 meses; $D P=2,9$ meses), e o peso de 3 a $10 \mathrm{~kg}$ (média: $6,3 \mathrm{~kg}$; $\mathrm{DP}=$ $1,8 \mathrm{~kg}$ ). Esses pacientes foram divididos em dois grupos (Grupos I e II) de acordo com o tipo de operação realizada: ou a paliação pela técnica de Blalock-Taussig ou a correção definitiva com circulação extracorpórea (CEC).

Grupo I - Entre março de 1986 e março de 1996, 26 crianças foram submetidas a operação de Blalock-Taussig (Tabela 1$)$. Vinte $(76,9 \%)$ eram do sexo masculino e $6(23,1 \%)$, do feminino. A idade variou de um a 10 meses (média: 5,3 meses; $\mathrm{DP}=$ 2,7 meses), e o peso de 3 a 9,4 kg (média: 5,8 kg; $\mathrm{DP}=1,8 \mathrm{~kg}$ ).

Todas as crianças apresentavam cianose em repouso e história de crises cianóticas. O exame clínico, eletrocardiograma e radiografia de tórax su- geriram o diagnóstico de TF, que foi confirmado, em todos os casos, pela ecodopplercardiografia. Contudo, em 9 pacientes, realizou-se cineangiocardiografia para melhor avaliação da anatomia das artérias pulmonares.

A operação de Blalock-Taussig foi sempre feita através de toracotomia póstero-lateral no lado contrário ao arco aórtico. Em 13 casos procedeu-se à operação clássica e em 13, ao Blalock modificado com a utilização de um enxerto tubular de Gore-Tex.

Nesse grupo de doentes procurou-se proceder à extubação na sala de operação, desde que tivessem padrão respiratório e gasometria adequadas, e nenhuma medida especial foi adotada no pós-operatório.

Grupo II - Entre janeiro de 1996 e setembro de 1999, 30 crianças com menos de um ano de idade portadoras de TF foram eletivamente submetidas a correção intracardíaca com CEC (Tabela 2). Dezesseis $(53,3 \%)$ eram do sexo masculino e $14(46,7 \%)$, do feminino. A idade variou de 3 a 11 meses (média: 7,3 meses; $\mathrm{DP}=2,7$ meses) e o peso entre $4 \mathrm{e}$ $10 \mathrm{~kg}$ (média: $7,1 \mathrm{~kg} ; \mathrm{DP}=1,4 \mathrm{~kg}$ ).

Vinte crianças tinham sintomas de hipoxemia e

TABELA 1

\begin{tabular}{|c|c|c|c|c|c|c|}
\hline \multicolumn{7}{|c|}{ OPERAÇÃO DE BLALOCK-TAUSSIG } \\
\hline CASO & No $\mathrm{FICHA}$ & INICIAIS & SEXO & $\begin{array}{l}\text { IDADE } \\
\text { (MESES) }\end{array}$ & $\begin{array}{l}\text { PESO } \\
(k g)\end{array}$ & $\begin{array}{c}\text { DATA } \\
\text { OPERAÇÃO }\end{array}$ \\
\hline 1 & 32 & EDN & M & 5 & 5,3 & 11.03 .86 \\
\hline 2 & 332 & ACCS & M & 7 & 6,2 & 09.07 .87 \\
\hline 3 & 514 & $J G L$ & M & 8 & 7,5 & 01.02 .88 \\
\hline 4 & 743 & EMS & M & 6 & 5,3 & 18.11 .88 \\
\hline 5 & 762 & AAL & $\mathrm{M}$ & 7 & 5,1 & 05.12 .88 \\
\hline 6 & 1120 & GCCS & $\mathrm{F}$ & 3 & 4,7 & 30.11 .89 \\
\hline 7 & 1179 & IAV & $\mathrm{M}$ & 7 & 6,3 & 06.02 .90 \\
\hline 8 & 1186 & AMT & $M$ & 4 & 4,5 & 16.02 .90 \\
\hline 9 & 1289 & WMS & $\mathrm{M}$ & 6 & 5,6 & 24.05 .90 \\
\hline 10 & 1361 & LV & M & 2 & 3,0 & 03.08 .90 \\
\hline 11 & 1374 & ASLJ & $\mathrm{M}$ & 4 & 4,7 & 10.08 .90 \\
\hline 12 & 472 & AASJ & $M$ & 9 & 8,7 & 11.09 .90 \\
\hline 13 & 859 & EMS & $\mathrm{F}$ & 2 & 4,3 & 28.09 .90 \\
\hline 14 & 1686 & MJS & M & 4 & 5,5 & 30.07 .91 \\
\hline 15 & 2054 & $\mathrm{RN} \mathrm{MCC}$ & M & 3 & 4,5 & 22.04 .92 \\
\hline 16 & 2139 & KDSS & M & 8 & 8,3 & 08.07.92 \\
\hline 17 & 2171 & LHFS & $M$ & 2 & 4,0 & 03.08 .92 \\
\hline 18 & 2294 & $\mathrm{FPL}$ & $M$ & 5 & 6,2 & 17.11 .92 \\
\hline 19 & 2452 & EDSS & M & 3 & 4,4 & 29.03 .93 \\
\hline 20 & 2716 & CPS & $\mathrm{F}$ & 10 & 9,0 & 01.11 .93 \\
\hline 21 & 2884 & EMS & $M$ & 4 & 5,4 & 04.04 .94 \\
\hline 22 & 3425 & DMMS & $\mathrm{F}$ & 10 & 9,3 & 24.05 .95 \\
\hline 23 & 3501 & LAM & $\mathrm{F}$ & 10 & 9,4 & 30.06 .95 \\
\hline 24 & 3658 & AMMA & $\mathrm{F}$ & 5 & 6,0 & 24.10 .95 \\
\hline 25 & 3682 & MAS & $M$ & 2 & 4,6 & 27.10 .95 \\
\hline 26 & 3887 & RLLS & $\mathrm{M}$ & 1 & 3,0 & 19.03 .96 \\
\hline
\end{tabular}


Moraes Neto F, Gomes C A, Lapa C, Hazin S, Tenório E, Mattos S, Moraes C R - Tratamento cirúrgico da tetralogia de Fallot no primeiro ano de vida. Rev Bras Cir Cardiovasc 2000; 15 (2): 143-53.

TABELA 2

\begin{tabular}{|c|c|c|c|c|c|c|}
\hline \multicolumn{7}{|c|}{ GRUPO II - CORREÇÃO TOTAL } \\
\hline CASO & No $\mathrm{FICHA}$ & INICIAIS & SEXO & $\begin{array}{l}\text { IDADE } \\
\text { (MESES) }\end{array}$ & $\begin{array}{c}\text { PESO } \\
(k g)\end{array}$ & $\begin{array}{c}\text { DATA } \\
\text { OPERAÇÃO }\end{array}$ \\
\hline 27 & 3775 & $\mathrm{RHA}$ & M & 11 & 9,0 & 15.01 .96 \\
\hline 28 & 3817 & LMM & M & 3 & 6,5 & 30.01 .96 \\
\hline 29 & 3894 & TMAO & $\mathrm{F}$ & 11 & 9,0 & 01.04 .96 \\
\hline 30 & 4103 & IMOF & $\mathrm{F}$ & 3 & 6,0 & 01.08 .96 \\
\hline 31 & 4106 & VMSS & $\mathrm{F}$ & 9 & 8,7 & 08.08 .96 \\
\hline 32 & 4112 & IVF & $\mathrm{F}$ & 6 & 6,0 & 12.08 .96 \\
\hline 33 & 4119 & AVSB & $\mathrm{F}$ & 8 & 7,0 & 29.08 .96 \\
\hline 34 & 4162 & $R G B$ & $\mathrm{M}$ & 8 & 8,0 & 09.09 .96 \\
\hline 35 & 4209 & LFAB & $M$ & 6 & 9,0 & 01.10 .96 \\
\hline 36 & 4231 & LHGA & $M$ & 7 & 8,0 & 21.10 .96 \\
\hline 37 & 4281 & ESF & $\mathrm{M}$ & 7 & 8,0 & 14.11 .96 \\
\hline 38 & 4329 & FFS & $\mathrm{M}$ & 10 & 6,0 & 18.12 .96 \\
\hline 39 & 4424 & JMBS & $\mathrm{M}$ & 4 & 6,0 & 06.03 .97 \\
\hline 40 & 4592 & DJCJ & $M$ & 9 & 9,0 & 18.06 .97 \\
\hline 41 & 4643 & WVS & M & 6 & 7,0 & 28.07 .97 \\
\hline 42 & 4814 & WSB & M & 10 & 7,0 & 28.10 .97 \\
\hline 43 & 4822 & LAL & $\mathrm{F}$ & 11 & 7,5 & 03.11 .97 \\
\hline 44 & 4917 & DEB & $\mathrm{F}$ & 4 & 4,0 & 16.01 .98 \\
\hline 45 & 4952 & NDS & $\mathrm{F}$ & 8 & 5,0 & 03.02 .98 \\
\hline 46 & 5144 & CML & $\mathrm{F}$ & 9 & 6,6 & 15.06 .98 \\
\hline 47 & 5275 & $A B C$ & $\mathrm{M}$ & 4 & 6,5 & 06.10 .98 \\
\hline 48 & 5317 & AGM & $\mathrm{F}$ & 7 & 10,0 & 04.11 .98 \\
\hline 49 & 5532 & ALB & $\mathrm{F}$ & 9 & 7,2 & 23.03 .99 \\
\hline 50 & 5644 & MSV & $M$ & 8 & 8,7 & 17.05 .99 \\
\hline 51 & 5650 & JLG & M & 1 & 4,0 & 27.05 .99 \\
\hline 52 & 5678 & $\mathrm{MHA}$ & $\mathrm{F}$ & 6 & 7,0 & 08.06.99 \\
\hline 53 & 5697 & GCS & $\mathrm{M}$ & 7 & 7,0 & 17.06 .99 \\
\hline 54 & 5719 & MIS & $\mathrm{F}$ & 5 & 7,0 & 01.07 .99 \\
\hline 55 & 5817 & RPSB & $M$ & 11 & 7,0 & 26.08 .99 \\
\hline 56 & 5844 & $\mathrm{GCG}$ & $\mathrm{F}$ & 11 & 7,0 & 02.09.99 \\
\hline
\end{tabular}

10 eram acianóticas e assintomáticas, e os achados clínicos, eletrocardiográficos e radiológicos eram sugestivos de TF. O diagnóstico definitivo foi estabelecido em todos os casos por ecodopplercardiografia bidimensional, tendo apenas uma criança sido submetida a cateterismo cardíaco em outro hospital.

Todos os pacientes foram operados através de esternotomia mediana com CEC e oxigenador de membranas. Em 29 utilizou-se hipotermia moderada $\left(25^{\circ} \mathrm{C}\right)$ e, em uma criança de apenas um mês e $4 \mathrm{~kg}$, hipotermia profunda e parada circulatória total. Obteve-se proteção miocárdica por infusão na aorta de solução cardioplégica cristalóide gelada e hipotermia tópica do coração.

A correção intracardíaca foi feita por via transventricular em 28 casos e por via atriopulmonar em 2 crianças cujo ramo interventricular anterior cruzava a via de saída do ventrículo direito (VD). Procedeu-se à ressecção de ambos os ramos da crista supraventricular e, nos casos de hipoplasia do anel pulmonar, realizou-se a ampliação transanular da via de saída do VD. O fechamento da comunicação interventricular (CIV) e a ampliação da via de saída do VD foram sempre feitas com pericárdio bovino em sutura contínua de Prolene 6-0.

Uma vez interrompida a CEC e obtida estabilidade hemodinâmica, realizaram-se, em 25 casos, medidas de pressão nas cavidades cardíacas direitas e colheram-se amostras de sangue para gasometria.

$\mathrm{Na}$ sala de recuperação, todas as crianças foram mantidas em assistência ventilatória por algumas horas com respirador de volume. Procurou-se realizar a extubação precocemente, desde que as condições hemodinâmicas fossem estáveis e não houvesse sangramento ou déficit neurológico.

Análise Estatística - Em ambos os grupos foram realizadas as estatísticas descritivas usuais, quais sejam média e desvio padrão (DP) para as variáveis contínuas e porcentagem para as variáveis categóricas. 
Moraes Neto F, Gomes C A, Lapa C, Hazin S, Tenório E, Mattos S, Moraes C R - Tratamento cirúrgico da tetralogia de Fallot no primeiro ano de vida. Rev Bras Cir Cardiovasc 2000; 15 (2): 143-53.

Utilizou-se o teste exato de Fisher para avaliar se houve diferença estatisticamente significante das mortalidades nos grupos I e II, adotando-se um nível de significância de $5 \%$.

\section{RESULTADOS}

\section{Grupo I}

Ocorreram $2(7,6 \%)$ óbitos no grupo de 26 crianças submetidas a operação de Blalock-Taussig. Uma (caso 2) faleceu no $5^{\circ}$ dia de pós-operatório, devido a insuficiência respiratória. A outra (caso 15) desenvolveu quadro de pulmão de choque, provavelmente por excessivo aumento do fluxo pulmonar após o shunt, vindo a falecer no $2^{\circ}$ dia de pósoperatório.
Todos os outros doentes tiveram alta hospitalar com apreciável melhora da cianose e nenhum exibiu complicações pós-operatórias.

No pós-operatório tardio, uma criança (caso 13) voltou a apresentar cianose intensa e foi submetida a outra operação de Blalock-Taussig, tendo falecido por complicações respiratórias. Seis pacientes (casos 4, 7, 8, 19, 20 e 22) já foram submetidos com sucesso a correção definitiva. Não ocorreram maiores dificuldades técnicas pela presença do shunt e, em 4 desses casos, foi necessário ampliar o anel pulmonar.

\section{Grupo II}

Dados Operatórios: Os principais dados transoperatórios de cada caso podem ser analisados na Tabela 3. O tempo de CEC variou de 50 a 125

TABELA 3

\begin{tabular}{|c|c|c|c|c|c|}
\hline \multicolumn{6}{|c|}{ GRUPO II - CORREÇÃO TOTAL } \\
\hline CASO & $\begin{array}{c}\text { AMPLIAÇÃO } \\
\text { VIA DE SAIDA VD }\end{array}$ & $\begin{array}{c}\text { TEMPO CEC } \\
(M I N)\end{array}$ & $\begin{array}{c}\text { TEMPO CAO } \\
\text { (MIN) }\end{array}$ & $\begin{array}{c}\text { SHUNT } \\
\text { RESIDUAL }\end{array}$ & $\begin{array}{c}\text { GRADIENTE } \\
\text { VD-TP }(\mathrm{mmHg})\end{array}$ \\
\hline 27 & VD & 90 & 63 & - & - \\
\hline 28 & - & 50 & 32 & - & 10 \\
\hline 29 & Transanular & 87 & 62 & - & - \\
\hline 30 & Transanular & 103 & 65 & - & 5 \\
\hline 31 & Transanular & 70 & 47 & - & 11 \\
\hline 32 & VD & 80 & 60 & - & 2 \\
\hline 33 & VD & 94 & 62 & - & 10 \\
\hline 34 & VD & 77 & 55 & - & 10 \\
\hline 35 & VD & 70 & 41 & - & 20 \\
\hline 36 & - & 65 & 45 & - & 10 \\
\hline 37 & Transanular & 72 & 46 & - & 11 \\
\hline 38 & Transanular & 105 & 67 & - & 25 \\
\hline 39 & VD & 73 & 56 & - & 12 \\
\hline 40 & - & 72 & 65 & - & 25 \\
\hline 41 & VD & 125 & 86 & - & 5 \\
\hline 42 & Transanular & 93 & 50 & - & 1 \\
\hline 43 & - & 60 & 38 & - & 7 \\
\hline 44 & Transanular & 125 & 60 & - & - \\
\hline 45 & - & 60 & 37 & - & 4 \\
\hline 46 & Trasanular & 91 & 60 & - & 28 \\
\hline 47 & - & 63 & 35 & - & - \\
\hline 48 & Transanular & 69 & 40 & - & - \\
\hline 49 & Transanular & 96 & 70 & - & 0 \\
\hline 50 & Transanular & 60 & 50 & - & 5 \\
\hline 51 & Transanular & $108^{*}$ & $52^{*}$ & - & 12 \\
\hline 52 & Transanular & 75 & 45 & - & 9 \\
\hline 53 & - & 70 & 45 & - & 10 \\
\hline 54 & Transanular & 75 & 48 & - & 13 \\
\hline 55 & - & 92 & 40 & - & 15 \\
\hline 56 & Transanular & 93 & 68 & - & 15 \\
\hline
\end{tabular}

* Hipotermia profunda e parada circulatória $(40 \mathrm{~min})$. CEC = circulação extracorpórea; CAo = clampeamento aórtico; VD = ventrículo direito; TP = tronco pulmonar; Ventr. = ventriculotomia. 
Moraes Neto F, Gomes C A, Lapa C, Hazin S, Tenório E, Mattos S, Moraes C R - Tratamento cirúrgico da tetralogia de Fallot no primeiro ano de vida. Rev Bras Cir Cardiovasc 2000; 15 (2): 143-53.

minutos (média: $82 \mathrm{~min}$; $\mathrm{DP}=19 \mathrm{~min}$ ) e o de pinçamento aórtico de 32 a 88 minutos (média: 53 min; $\mathrm{DP}=13 \mathrm{~min})$. Em 22 pacientes (73,3\% dos casos), realizou-se ampliação da via de saída do VD, sendo que, em 15 (50\%), a ampliação se estendeu ao anel e tronco pulmonar. Em nenhum paciente detectou-se, ao final da operação, shunt residual. Em 25 casos nos quais se mediram as pressões ao final da operação, o gradiente pressórico entre o VD e a artéria pulmonar variou de 0 a $28 \mathrm{mmHg}$ (média: $11 \mathrm{mmHg}$ ).

Pós-Operatório Imediato: Ocorreram 2 óbitos $(6,6 \%)$ nesta série de pacientes (casos 44 e 53), ambos em decorrência de insuficiência renal. A grande maioria dos doentes teve evolução pós-operatória sem intercorrências, registrando-se apenas que uma criança exigiu ventilação artificial prolongada (mais de 12 horas) e uma outra apresentou parada cardíaca de fácil reversão devido a hiperpotassemia. Dezesseis pacientes exibiram, no pós-operatório, sinais de insuficiência cardíaca congestiva, sendo necessário o uso de digital, diuréticos e vasodilatadores.

Pós-Operatório Tardio: Uma criança faleceu sete meses após a operação, em decorrência de meningite (caso 37). Todos os outros apresentam excelente evolução clínica e encontram-se assintomáticos. O estudo ecocardiográfico pós-operatório tem evidenciado função ventricular normal, em todos os casos, e gradientes pouco significativos $(<40 \mathrm{mmHg})$ na via de saída do VD.

\section{Análise Estatística}

O resultado do teste exato de Fisher mostra que ao nível de $5 \%$ a mortalidade no Grupo I (7,6\%) não foi, do ponto de vista estatístico, siginificantemente diferente da mortalidade de $6,7 \%$ do Grupo II $(p=1,00)$.

\section{COMENTÁRIOS}

O tratamento tradicional de crianças sintomáticas com TF no primeiro ano de vida ainda é, na maioria dos Centros de Cirurgia Cardíaca, a paliação através da operação de Blalock-Taussig. Muitos advogam essa conduta pelo baixo risco que ela apresenta. Ademais, a maioria das crianças exibe imediata melhora clínica e não se observam efeitos desfavoráveis quando da correção total (1-8).

NECHES et al. (9) e ARCINIEGAS et al. (10) demonstraram que a operação de Blalock-Taussig tem menor morbidade e mortalidade que outras anastomoses sistêmico-pulmonares. TYSON et al. (2) não tiveram mortalidade e complicações significativas em 12 crianças com menos de um ano de idade submetidas a operação de Blalock-Taussig. GUYTON et. al. (5) também não observaram mortalidade em 19 casos de TF no primeiro ano de vida submetidos a operação de Blalock-Taussig e demonstraram, com estudos angiográficos pós-operatórios, o crescimento de ambos os ramos da artéria pulmonar. ILBAWI et al. (7) utilizaram o Blalock modificado numa série de 30 neonatos com idade média de 8,8 dias, 10 dos quais tinham TF. Nestes últimos não se observou mortalidade, trombose do shunt ou distorção das artérias pulmonares, embora 2 pacientes tenham necessitado outra operação do lado oposto por aumento da cianose. Na Universidade do Alabama (11-12), também não houve mortalidade com esse tipo de procedimento e muito bons resultados também foram descritos, entre outros, por STEPHENSON et al. (1), ARCINIEGAS et al. (3), KAY et al. (4), LAMBERTI et al. (6) e BOVE et al. (8). Em nossa série de 26 crianças com menos de um ano portadoras de TF e submetidas a operação de Blalock-Taussig, a mortalidade foi de 7,6\% (2 óbitos), maior, portanto, do que a relatada nas séries citadas, mas bastante aceitável, levando-se em conta a gravidade desse grupo de doentes.

No momento, há uma tendência, na maioria dos Centros, de se realizar a técnica do Blalock modificado $(4,7)$. Em metade dos nossos casos, essa técnica foi utilizada e concordamos com a opinião de que ela simplifica a operação. Há também evidência de que a utilização do Gore-Tex em neonatos diminui a incidência de trombose da anastomose (13). Essa complicação, que ocorre em aproximadamente 7\% dos casos no primeiro mês após a operação, não foi observada em nosso grupo de doentes.

O fechamento ou a estenose tardia do shunt varia de 3 a $20 \%$ em diversas séries relatadas e parece ser mais freqüente quando a operação é realizada em neonatos $(4,6,14-16)$. Em nossa experiência, isso ocorreu em apenas uma criança $(3,8 \%)$, submetido a um novo shunt seis meses depois da primeira operação.

Alterações iatrogênicas das artérias pulmonares, observadas com freqüência após a operação de Waterston, são raras após a operação de BlalockTaussig $(8,11)$ e não foram observadas em nossa casuística. Também não observamos efeitos adversos nos seis casos já submetidos, com sucesso, a correção total.

$\mathrm{Na}$ evolução tardia de crianças submetidas a operação de Blalock-Taussig verifica-se, na maioria dos casos, que em decorrência do aumento do fluxo sangüíneo pulmonar, ocorre redução da cianose e da policitemia e melhora da capacidade funcional. Contudo, é importante recordar que esses benefícios são obtidos à custa de um aumento do traba- 
Moraes Neto F, Gomes C A, Lapa C, Hazin S, Tenório E, Mattos S, Moraes C R - Tratamento cirúrgico da tetralogia de Fallot no primeiro ano de vida. Rev Bras Cir Cardiovasc 2000; 15 (2): 143-53.

Iho do ventrículo esquerdo, o que pode gradualmente induzir à disfunção ventricular.

Outro benefício decorrente da operação de Blalock-Taussig é promover um difuso aumento no tamanho dos ramos e tronco da artéria pulmonar, e mesmo do anel, e subseqüentemente diminuir a necessidade de ampliação da via de saída do VD (17). Esse, que seria, em nossa opinião, o melhor argumento dos que defendem a paliação inicial não foi provado até hoje. Em nossa experiência, dos 6 pacientes já submetidos a correção total, após operação de Blalock-Taussig, 4 (6,6\%) necessitaram ampliação trasanular da via de saída do VD. Idêntica observação foi feita por SADE et al. (18).

$O$ fato da TF poder ser corrigida com CEC no primeiro ano de vida com baixas morbidade e mortalidade foi demonstrado por BARRATT-BOYES \& NEUTZE (19) e STARR et al. (20), em 1973. Também nessa ocasião consideraram ser improvável que os resultados tardios fossem diferentes dos obtidos em crianças maiores. Essa conduta foi igualmente adotada por outros grupos (21-30).

As possíveis vantagens da correção nos primeiros meses de vida incluiriam: 1) normalização precoce do fluxo e das pressões em todas as câmaras cardíacas; 2) interrupção do processo de hipertrofia do VD, que ocorre quando essa cavidade trabalha na presença de estenose pulmonar; 3) ressecção menor do infundíbulo, admitindo-se que isso leva a uma diminuição da incidência tardia de arritmias ventriculares; 4) normalização precoce da saturação arterial de oxigênio, evitando-se os efeitos deletérios da hipoxemia crônica sobre outros órgãos; 5) evitar potenciais complicações das operações de shunt, especialmente distorção das artérias pulmonares e desenvolvimento de hipertensão pulmonar; 6) finalmente, há nítidas vantagens econômicas e psicossociais.

Evidentemente, esses atraentes argumentos precisaram ser comprovados com experiência clínica maior, demonstrando baixas mortalidade e morbidade imediatas e bons resultados tardios. Nesse sentido, o grupo do Children's Hospital, de Boston, deu uma grande contribuição. CASTANEDA et al. (22), em 1977, relataram os primeiros 41 casos consecutivos de TF, operados no primeiro ano de vida, com hipotermia profunda e parada circulatória. Em $70 \%$ dos casos, foi necessária a ampliação do anel pulmonar. A mortalidade hospitalar foi de 7\% (3 casos). Dezenove pacientes foram reestudados hemodinamicamente um ano após a operação, constatando-se 2 casos de aneurisma do VD e 1 de comunicação interventricular residual, reoperados com sucesso. DIDONATO et al. (31), do mesmo grupo, relataram em 1989 os resultados da correção definitiva em 26 neonatos (idade média de 8,2 dias), 12 dos quais tinham atresia pulmonar com 5 óbitos. CASTANEDA (32) relatou que, entre 1973 e 1993, 330 casos de TF foram operados no primeiro ano de vida com 14 óbitos (4,2\%) hospitalares e 3 tardios $(0,9 \%)$.

Esses excelentes resultados do grupo de Boston foram reproduzidos em outros Centros. DAILY et al. (33), da Stanford, analisaram vários fatores de risco na operação de TF e concluíram que a correção precoce é melhor, pois a mortalidade foi de $5 \%$, exceto nos casos com grave hipoplasia dos ramos da artéria pulmonar. TUCKER et al. (23), de San Francisco, relataram 21 casos de correção em crianças menores de um ano com 3 óbitos; GUSTAFSON et al. (24), de West Virginia, 10 casos sem óbitos; TOUATI et al. (25), do Hospital Laennec, de Paris, 100 casos com $3 \%$ da mortalidade; GROH et al. (26), 58 casos sem mortalidade, operados nas Universidades de Michigan e do Alabama; SOUSA UVA et al. (27), do Hospital Marie Lannelongue, de Paris, 41 casos com 1 óbito; HENNEIN et al. (34), de Michigan, 30 neonatos, com idade média de 11 dias, sem óbitos; REDDY et al. (30), de San Francisco, 30 neonatos sem mortalidade; e STELLIN et al. (29), da Universidade de Pádua, Itália, 51 casos com menos de seis meses de vida e apenas 1 óbito. Nossa experiência preliminar repetiu esses resultados (35). Mesmo sem fazermos seleção de doentes (todas as crianças que chegaram ao Serviço após março de 1996, com diagnóstico de TF, tiveram indicação para correção definitiva), obtivemos excelentes resultados com baixa morbidade e mortalidade de $6,6 \%$ (2 casos), estatisticamente semelhante à mortalidade que obtivemos com a operação de Blalock-Taussig $(7,6 \%)$. Evidentemente, a decisão de corrigir a TF no primeiro ano de vida pressupõe que o grupo cirúrgico tenha grande experiência no manuseio dessa malformação e disponha de condições adequadas. Do contrário, é mais prudente adotar-se a conduta da correção da TF em dois tempos (36).

Um dos aspectos mais controversos da correção da TF no primeiro ano de vida relaciona-se à necessidade de ampliação transanular da via de saída do VD. VOBECKY et al. (37), analisando a sobrevivência de pacientes com TF, operados no Hospital for Sick Children, de Toronto, consideram que a correção primária precoce parece proteger a função neurológica, a função ventricular esquerda e produzir menos arritmias. Entretanto, há maior incidência da necessidade de patch transanular, o que pode comprometer a função do VD no pósoperatório tardio. KIRKLIN et al. (38) consideram que a colocação de patch transanular é um importante fator de risco em crianças com superfície corporal $<0,48 \mathrm{~m}^{2}$. A análise de diversas séries mostra que a incidência de patch transanular varia de 30 a $70 \%$. Na nossa experiência, foi de $50 \%$. Em nossa 
Moraes Neto F, Gomes C A, Lapa C, Hazin S, Tenório E, Mattos S, Moraes C R - Tratamento cirúrgico da tetralogia de Fallot no primeiro ano de vida. Rev Bras Cir Cardiovasc 2000; 15 (2): 143-53.

opinião, essa maior incidência na necessidade de ampliação do anel pode refletir a gravidade da morfologia dos casos que têm sido operados no primeiro ano de vida, pois a maioria dos grupos somente tem indicado a operação em crianças muito sintomáticas. Reforça essa idéia o fato, já mencionado, de que as crianças paliadas no primeiro ano de vida também necessitam, em uma alta incidência, ampliação do anel durante a correção definitiva.

CASTANEDA et al. (22) estabeleceram que os dois únicos fatores de risco que contra-indicam a correção no primeiro ano de vida são hipoplasia acentuada das artérias pulmonares e origem anômala da artéria descendente anterior da coronária direita. GROH et al. (26) e, mais recentemente, REDDY et al. (30) consideram que a operação não deve ser contra-indicada com base apenas no tamanho das artérias pulmonares, continuando este um aspecto ainda aberto à discussão. Com relação à anomalia de origem da artéria descendente anterior, isso pode ser obviado pela adoção da técnica de correção transatrial-transpulmonar como em 2 de nossos pacientes.

Os resultados tardios da correção da TF no primeiro ano de vida são excelentes e comparáveis aos observados em crianças maiores (39-45). CAL-
DER et al. (39) analisaram os dados hemodinâmicos e radiológicos em 23 crianças operadas no primeiro ano de vida e verificaram resultados bons ou excelentes em $96 \%$ dos casos. BOROW et al. (40), utilizando sofisticados métodos da avaliação da função ventricular esquerda, demonstraram ser esta melhor preservada nas crianças submetidas à correção precocemente. SELIEM et al. (42) demonstraram que a regressão da hipertrofia ventricular direita possui relação direta com a precocidade da operação.

A evolução clínica tardia dos nossos pacientes é compatível com os dados da literatura. Todos têm excelente situação clínica, e a avaliação ecocardiográfica não mostra CIV residual, gradiente significativo na via de saída do VD e a função ventricular é normal.

Acreditamos, com base em dados da literatura e, especialmente, na experiência relatada no presente trabalho, que a operação de escolha em pacientes com TF clássica no primeiro ano de vida é a correção intracardíaca primária, assumindo que o grupo cirúrgico tenha experiência no manuseio de pequenas crianças com cardiopatias complexas e disponha de acurados métodos de diagnóstico e de excelentes cuidados de pósoperatório.

RBCCV 44205-496

Moraes Neto F, Gomes C A, Lapa C, Hazin S, Tenório E, Mattos S, Moraes C R - Surgical treatment of tetralogy of Fallot in the first year of life. Rev Bras Cir Cardiovasc 2000; 15 (2): 143-53.

ABSTRACT: Objective: To analysis of morbidity and mortality of surgical treatment of the classic of tetralogy of Fallot in the first year of life and particularly to define possible advantages of early primary repair.

Material and Methods: Between March 1986 and September 1999, 56 children under one age tetralogy of Fallot underwent surgical treatment. Thirty-six $(64.3 \%)$ were male and $20(35.7 \%)$ female, ranging in age from 1 to 11 months (mean $6.5 \pm 2.9$ mo.). Weight ranged from 3 to $10 \mathrm{~kg}$ (mean $6.3 \pm$ $1.8 \mathrm{~kg}$ ). The patients were divided into 2 groups: Group I, consisted of 26 children operated on between 1986 and 1996, submitted to a Blalock-Taussig shunt; Group II, comprised of 30 children operated on consecutively since 1996 and submitted to intracardiac repair.

Results: In Group I, there were $2(7.6 \%)$ early and $1(3.8 \%)$ late deaths. No postoperative complications were observed in the remaining children. In Group II, 2 (6.6\%) early deaths and 1 (3.3\%) noncardiac late death of a have occurred. Only 2 children had non-significant postoperative complications and 16 presented signs of congestive heart failure. The mortality in both groups was not statistically significant.

Conclusions: In the authors' experience, ideal management of children with classical Fallot's tetralogy in the first year of life consists of the intracardiac repair since it has the same surgical risk as palliation.

DESCRIPTORS: Tetralogy of Fallot, surgery. Cardiac surgical procedures, pediatric 
Moraes Neto F, Gomes C A, Lapa C, Hazin S, Tenório E, Mattos S, Moraes C R - Tratamento cirúrgico da tetralogia de Fallot no primeiro ano de vida. Rev Bras Cir Cardiovasc 2000; 15 (2): 143-53.

\section{REFERÊNCIAS BIBLIOGRÁFICAS}

1 Stephenson L W, Friedman S, Edmunds LH Jr. Staged surgical management of tetralogy of Fallot in infants. Circulation 1978; 58: 837-41.

2 Tyson $\mathrm{K}$ R, Larrieu A J, Kirchmer J T Jr. - The Blalock-Taussig shunt in the first two years of life: a safe and effective procedure. Ann Thorac Surg 1978; 26: 38-41.

3 Arciniegas E, Blackstone E H, Pacifico A D, Kirklin J W - Classic shunting operations as part of twostage repair of tetralogy of Fallot. Ann Thorac Surg 1979; 27: 514-8.

4 Kay $\mathrm{P} \mathrm{H}$, Capuani A, Franks R, Lincoln C - Experience with the modified Blalock-Taussig operation using polytetrafluoroethylene (Impra) grafts. $\mathrm{Br}$ Heart J 1983; 49: 359-63.

5 Guyton R A, Owens J E, Waumett J D, Dooley K J, Hatcher C R Jr., Williams W H - The BlalockTaussig shunt: low risk, effective palliation, and pulmonary artery growth. J Thorac Surg 1983; 85: 917-22.

6 Lamberti J J, Carlisle J, Waldman J D et al. - Systemic-pulmonary shunts in infants and children: early and late results. J Thorac Cardiovasc Surg 1984; 88(5 Pt 1): 76-81.

7 Ilbawi M N, Grieco J, DeLeon S Y et al. - Modified Blalock-Taussig shunt in newborn infants. $J$ Thorac Cardiovasc Surg 1984; 88: 770-5.

8 Bove E L, Kohman L, Sereika S et al. - The modified Blalock-Taussig shunt: analysis of adequacy and duration of palliation. Circulation 1987; 76: (3 Pt 2): III19-23.

9 Neches W H, Naifeh J G, Park S C et al. - Systemicpulmonary artery anastomoses in infancy. $J$ Thorac Cardiovasc Surg 1975; 70: 921-7.

10 Arciniegas E, Farooki Z Q, Hakimi M, Perry B L, Green E W - Classic shunting operations for congenital cyanotic heart defects. J Thorac Cardiovasc Surg 1982; 84: 88-96.

11 Kirklin J W \& Barratt-Boyes B G - Tetralogy of Fallot with pulmonary stenosis. In: Kirklin J W \& BarrattBoyes B G, eds. Cardiac surgery. New York: Churchill Livingstone, 1993; 863-942.

12 Kirklin J W, Blackstone E H, Kirklin J K, Pacifico A $D$, Aramendi J, Bargeron L M Jr. - Surgical results and protocols in the spectrum of tetralogy of Fallot. Ann Surg 1983; 198: 251-65.

13 Ullom R L, Sade R M, Crawford F A Jr., Ross B A, Spinale $\mathrm{F}$ - The Blalock-Taussig shunt in infants: standard versus modified. Ann Thorac Surg 1987; 44: 539-43.

14 Donahoo J S, Gardner T J, Zahka K, Kidd B S -
Systemic-pulmonary shunts in neonates and infants using microporous expanded polytetrafluoroethylene: immediate and late results. Ann Thorac Surg 1980; 30: 146-50.

15 Di Benedetto G, Tiraboschi R, Vanini $V$ et al. - Systemic-pulmonary artery shunt using PTFE prosthesis (Gore-Tex): early results and long-term follow-up on 105 consecutive cases. Thorac Cardiovasc Surg 1981; 29: 143-7.

16 McKay R, de Leval M R, Rees P, Taylor J F, Macartney $\mathrm{F} J$, Stark J - Postoperative angiographic assessment of modified Blalock-Taussig shunts using expanded polytetrafluoroethylene (GoreTex). Ann Thorac Surg 1980; 30: 137-45.

17 Villani M, Gamba A, Tiraboschi R, Crupi G, Parenzan $\mathrm{L}$ - Surgical treatment of tetralogy of Fallot: recent experience using a prospective protocol. Thorac Cardiovasc Surg 1983; 31: 151-5.

18 Sade R M, Sloss L, Treves S, Bernhard W F, Castaneda A R - Repair of tetralogy of Fallot after aortopulmonary anastomosis. Ann Thorac Surg 1977; 23: 32-8.

19 Barratt-Boyes BG \& Neutze JM - Primary repair of tetralogy of Fallot in infancy using profound hypothermia with circulatory arrest and limited cardiopulmonary bypass: a comparison with conventional two-stage management. Ann Surg 1973; 178: 406-11.

20 Starr A, Bonchek L I, Sunderland C O - Total correction of tetralogy of Fallot in infancy. J Thorac Cardiovasc Surg 1973; 65: 45-57.

21 Castaneda A R, Mayer J E Jr., Jonas R A, Lock J E, Wessel D L, Hickey P R - The neonate with critical congenital heart disease: repair, a surgical challenge. J Thorac Cardiovasc Surg 1989; 98 (5 Pt 2): 869-75.

22 Castaneda A R, Freed M D, Williams R G, Norwood W I Jr. - Repair of tetralogy of Fallot in infancy: early and late results. J Thorac Cardiovasc Surg 1977; 74: 372-81.

23 Tucker W Y, Turley K, Ullyot D J, Ebert P A Management of symptomatic tetralogy of Fallot in the first year of life. $J$ Thorac Cardiovasc Surg 1979; 78: 494-501.

24 Gustafson R A, Murray G F, Warden H E, Hill R C, Rozar G E Jr. - Early primary repair of tetralogy of Fallot. Ann Thorac Surg 1988; 45: 235-41.

25 Touati G D, Vouhé P R, Amodeo A et al. - Primary repair of tetralogy of Fallot in infancy. J Thorac Cardiovasc Surg 1990; 99: 396-403.

26 Groh M A, Meliones J N, Bove E L et al. - Repair of tetralogy of Fallot in infancy: effect of pulmonary artery size on outcome. Circulation 1991; 84 (5 Suppl): III206-12. 
Moraes Neto F, Gomes C A, Lapa C, Hazin S, Tenório E, Mattos S, Moraes C R - Tratamento cirúrgico da tetralogia de Fallot no primeiro ano de vida. Rev Bras Cir Cardiovasc 2000; 15 (2): 143-53. Surgery for tetralogy of Fallot at less than six months of age. J Thorac Cardiovasc Surg 1994; 107: $1291-300$.

Starnes V A, Luciani G B, Latter D A, Griffin M L Current surgical management of tetralogy of Fallot. Ann Thorac Surg 1994; 58: 211-5.

Stellin G, Milanesi O, Rubino M et al. - Repair of tetralogy of Fallot in the first six months of life: transatrial versus transventricular approach. Ann Thorac Surg 1995; 60 (6 Suppl): S588-91.

Reddy V M, Liddicoat J R, McElhinney D B, Brook M $M$, Stanger $P$, Hanley $F L-$ Routine primary repair of tetralogy of Fallot in neonates and infants less than three months of age. Ann Thorac Surg 1995; 60 (6 Suppl): S592-6.

DiDonato R, Jonas R A, Lang P, Rom J J, Mayer J E, Castaneda A R - Neonatal repair of Fallot's tetralogy with and without pulmonary atresia. $J$ Am Coll Cardiol 1989; 13 (Suppl): S74-81.

Castaneda AR - Invited commentary in Vobecky et al. (37).

Daily $\mathrm{P}$ O, Stinson E B, Griepp R B, Shumway N E - Tetralogy of Fallot: choice of surgical procedure. J Thorac Cardiovasc Surg 1978; 75: 338-45.

Hennein H A, Mosca R S, Urcelay G, Crowley D C, Bove $\mathrm{E} \mathrm{L} \mathrm{-} \mathrm{Intermediate} \mathrm{results} \mathrm{after} \mathrm{complete}$ repair of tetralogy of Fallot in neonates. J Thorac Cardiovasc Surg 1995; 109: 332-43.

Moraes Neto F, Lapa C, Moraes C R et al. - Correção total da tétrade de Fallot no primeiro ano de vida. Rev Bras Cir Cardiovasc 1998; 13: 29-32.

36 Atik E - Tetralogia de Fallot no neonato: correção operatória ou técnica paliativa? Arq Bras Cardiol 1997; 68: 393-5.

37 Vobecky S J, Williams W G, Trusler G A et al. Survival analysis of infants under age 18 months presenting with tetralogy of Fallot. Ann Thorac Surg 1993; 56: 944-50.

Kirklin J W, Blackstone E H, Pacifico A D, Brown R $\mathrm{N}$, Bargeron L M Jr. - Routine primary repair vs two-stage repair of tetralogy of Fallot. Circulation 1979; 60: 373-86

39 Calder A L, Barratt-Boyes B G, Brandt P W, Neutze $J M$ - Postoperative evaluation of patients with tetralogy of Fallot repaired in infancy: including criteria for use of outflow patching and radiologic assessment of pulmonary regurgitation. J Thorac Cardiovasc Surg 1979; 77: 704-20.

40 Borow K M, Green L H, Castaneda A R, Keane J F - Left ventricular function after repair of tetralogy of Fallot and its relationship to age at surgery. Circulation 1980; 61: 1150-8.
41 Walsh E P, Rockenmacher S, Keane J F, Hougen T $J$, Lock J E, Castaneda A $\mathrm{R}$ - Late results in patients with tetralogy of Fallot repaired during infancy. Circulation 1988; 77: 1062-7.

42 Seliem M A, Wu Y T, Glenwright K - Relation between age at surgery and regression of right ventricular hypertrophy in tetralogy of Fallot. Pediatr Cardiol 1995; 16: 53-5.

43 Finnegan $P$, Haider R, Patel R G, Abrams L S, Singh $S \mathrm{P}-$ Results of total correction of the tetralogy of Fallot: long-term haemodynamic evaluation at rest and during exercise. $\mathrm{Br}$ Heart $J$ 1976; 38: 934-42.

44 James F W, Kaplan S, Schwartz D C, Chou T C, Sandker M J, Naylor V - Response to exercise in patients after total surgical correction of tetralogy of Fallot. Circulation 1976; 54: 671-9.

45 Fuster V, McGoon D C, Kennedy M A, Ritter D G, Kirklin J W - Long-term evaluation (12 to 22 years) of open heart surgery for tetralogy of Fallot. $\mathrm{Am}$ J Cardiol 1980; 46: 635-42.

\section{Discussão (Transcrições de fita gravada)}

\section{DR. MAURO BARBOSA ARRUDA FILHO Recife, $P E$}

Agradeço à Comissão Organizadora deste Congresso o convite para comentar o belíssimo trabaIho desenvolvido pelo Dr. Fernando e por seu grupo, sobre o tratamento cirúrgico da tetralogia de Fallot no primeiro ano de vida. Como o Dr. Fernando frizou, o tema abordado não foi totalmente absorvido pela comunidade internacional e nacional, embora, aceito por um grande número de cirurgiões. A grande controvérsia é se a correção deve ser realizada em dois tempos ou em um único. Como a tetralogia de Fallot é uma cardiopatia que se acha associada a outras variáveis, como a atresia da artéria pulmonar e a hipoplasia de seus ramos, a cirurgia do shunt ainda é mandatária. Gostaria de saber qual a conduta do Dr. Fernando quando essas variáveis estão associadas? O seu trabalho também é contemporâneo de outros autores que aderiram à correção total na mesma faixa etária, porém utilizando a via atriopulmonar, por considerarem fator determinante da boa função ventricular direita. Em $93 \%$ da sua casuística foi utilizada a via transventricular, com acesso transverso e longitudinal; qual o critério dessa preferência? Em nosso Serviço, 16 pacientes desenvolveram insuficiência cardíaca congestiva no pós-operatório. É sabido que, apesar de não ser o objetivo do seu trabalho, a ausência de um sistema valvar competente transforme essa condição clínica de aguda para algum grau de cronificidade, sendo detectada somente ao longo 
Moraes Neto F, Gomes C A, Lapa C, Hazin S, Tenório E, Mattos S, Moraes C R - Tratamento cirúrgico da tetralogia de Fallot no primeiro ano de vida. Rev Bras Cir Cardiovasc 2000; 15 (2): 143-53.

dos anos com estudo de resposta ao trabalho. Apesar de seu seguimento ter apenas 4 anos, gostaria de ouvir a sua opinião sobre esse assunto. Mais uma vez cumprimento-o pela excelência de seu trabalho. Obrigado.

\section{DR. ARLINDO RISO}

\section{São Paulo, SP}

A correção da tetralogia de Fallot no primeiro ano de vida é um tema de grande interesse. Gostaria de saber se sua casuística é formada por pacientes operados consecutivamente? Na minha opinião, há uma gama de pacientes que, provavelmente, não se beneficiariam tanto com a correção total realizada precocemente, como os portadores de hipoplasia das artérias pulmonares ou com anel pulmonar pequeno? Eu vi na sua apresentação que $50 \%$ dos pacientes do grupo 2 tiveram ampliação do anel pulmonar, o que na literatura ocorre em torno de $70-75 \%$ dos casos. Gostaria de saber qual era a condição pré-operatória desses pacientes? Eu julgo que eram casos mais favoráveis para se fazer uma correção e não aqueles casos com extrema hipoplasia das artérias pulmonares ou do anel pulmonar. Outra coisa que você mencionou foi a ampliação do ventrículo direito, na sua correção por ventriculotomia, você faz sutura direta ou sempre coloca um patch na via de saída? Outro aspecto que eu gostaria de comentar é sobre a função ventricular; durante a sua apresentação, afirmou que esta era normal; eu entendi que é a função ventricular esquerda. Na tetralogia de Fallot, nós sabemos que a função do ventrículo esquerdo é normal, o que mais nos preocupa é a função ventricular direita em relação à insuficiência pulmonar, à ventriculotomia e à ampliação do anel. Atualmente, há um grande arsenal diagnóstico; entretanto, para avaliar a função do ventrículo direito ainda não há nenhum que seja tão fidedigno. Eu gostaria de saber a sua opinião sobre isso. Muito obrigado.

\section{DR. MARCELO B. JATENE \\ São Paulo, SP}

Eu gostaria de cumprimentar o Dr. Fernando, pelo trabalho que considero extremamente importante. Fiquei com algumas dúvidas, particularmente em como conduzir determinados grupos de crianças. A partir de que idade, dentro do primeiro ano de vida, você considera ideal a correção definitiva e em quais circunstâncias? Seria entre o $6^{\circ}$ mês e $01^{\circ}$ ano, ou abaixo do $6^{\circ}$ mês? Alguns de seus pacientes tinham 1 mês de vida, e eu suponho que todos eles tenham sido submetidos a Blalock, clássico ou modificado. Dando uma abordagem mais específica para a correção definitiva, a partir de que idade você manuseia essas crianças? Obrigado.

\section{DR. MORAES NETO \\ (Encerrando)}

Inicialmente, gostaria de esclarecer que a grande maioria dos pacientes operados nessa série foram de tetralogia de Fallot clássico, e não complicado. Com relação à via de abordagem, a preferencialmente adotada em nossa Instituição é a ventriculotomia direita; vários trabalhos têm mostrado que não existe diferença em relação ao pósoperatório imediato ou tardio. Além disso, nossa equipe já conta com a experiência de aproximadamente 400 casos adotando essa via de acesso, estando essa técnica bem estabelecida no grupo. Com relação à ampliação do ventrículo direito por via transanular, é verdade que a ampliação da via de saída estendendo-se para o anel e tronco da artéria pulmonar favorece no pós-operatório desses pacientes um manuseio mais difícil em decorrência da insuficiência pulmonar, conseqüentemente levando a insuficiência cardíaca no pós-operatório. Infelizmente, não há tempo para mostrar aqui, mas no período que estive no Brompton Hospital, tive a oportunidade de trabalhar com um grupo que estuda há vários anos a fisiologia do ventrículo direito. Em um dos trabalhos do qual participei observouse que varia a resposta de paciente para paciente com relação à insuficiência pulmonar. Alguns pacientes apresentam a fisiologia restritiva do ventrículo direito, o que favorece a evolução tardia; em contraposição eles têm um pós-operatório mais turbulento com insuficiência cardíaca mais acentuada. No nosso grupo, 1 paciente apresentava atresia pulmonar, o qual necessitou de intubação mais prolongada, com uma evolução mais arrastada. Com relação aos comentários do Dr. Arlindo, desde 1996 adotamos essa conduta na Instituição e, a partir de então, apenas 2 casos foram submetidos à operação de Blalock. Um desses pacientes apresentava atresia pulmonar, hipoplasia de ramos e síndrome de Down e o outro paciente apresentava vários problemas genéticos e, por isso, optamos por uma terapêutica menos agressiva. Realmente, tivemos, em nossa experiência, um índice menor de ampliação da via de saída do ventrículo direito, em 50\% de nossos pacientes. Isto depende, obviamente, da condição clínica do paciente; como a grande maioria desses pacientes apresentava tetralogia de Fallot clássica, não havia obstruções muito significativas. Com relação ao fechamento do ventrículo direito, sempre utilizamos a aplicação do patch para fechar a ventriculotomia direita. Com relação à função do ventrículo direito no pós-operatório, todos 
esses pacientes têm sido avaliados pela equipe da Cardiologia Pediátrica. É verdade que a função ventricular esquerda é normal, na grande maioria desses pacientes, e, em todos, eles têm obtido o desmame das drogas gradativamente. Respondendo à pergunta do Dr. Marcelo sobre quando indicar a correção da tetralogia de Fallot, a grande maioria dos nossos pacientes foi operada acima dos 3 meses de vida, apenas 1 paciente foi operado com 1 mês, pois tinha uma anatomia favorável para fazer a correção. A política na nossa Instituição é postergar até no máximo 3-6 meses, quando partimos para fazer a correção; no início, preferíamos em torno de 6 meses, esperando a criança ganhar um pouco de peso. Com o manuseio desse grupo de pacientes, estamos nos atrevendo a realizar cada vez mais precocemente essa correção. Obrigado pelos comentários. 